\title{
Training-Induced Neural Plasticity in Golf Novices
}

\author{
Ladina Bezzola, ${ }^{1,2}$ Susan Mérillat, ${ }^{2}$ Christian Gaser, ${ }^{3,4}$ and Lutz Jäncke ${ }^{1,2}$ \\ ${ }^{1}$ Institute of Psychology, Division Neuropsychology and ${ }^{2}$ International Normal Aging and Plasticity Imaging Center, University of Zurich, CH-8050 Zurich, \\ Switzerland, and ${ }^{3}$ Department of Psychiatry and ${ }^{4}$ Department of Neurology, University of Jena, D-07737 Jena, Germany
}

Previous neuroimaging studies in the field of motor learning have shown that learning a new skill induces specific changes of neural gray and white matter in human brain areas necessary to control the practiced task. Former longitudinal studies investigating motor skill learning have used strict training protocols with little ecological validity rather than physical leisure activities, although there are several retrospective and cross-sectional studies suggesting neuroprotective effects of physical leisure activities. In the present longitudinal MRI study, we used voxel-based morphometry to investigate training-induced gray matter changes in golf novices between the age of 40 and 60 years, an age period when an active life style is assumed to counteract cognitive decline. As a main result, we demonstrate that $40 \mathrm{~h}$ of golf practice, performed as a leisure activity with highly individual training protocols, are associated with gray matter increases in a task-relevant cortical network encompassing sensorimotor regions and areas belonging to the dorsal stream. A new and striking result is the relationship between training intensity (time needed to complete the 40 training hours) and structural changes observed in the parieto-occipital junction. Thus, we demonstrate that a physical leisure activity induces training-dependent changes in gray matter and assume that a strict and controlled training protocol is not mandatory for training-induced adaptations of gray matter.

\section{Introduction}

The human brain demonstrates an enormous capacity to adapt quickly and efficiently to a broad variety of environmental demands. Among others, skill acquisition and training in various domains, such as motor function or cognition, were shown to evoke substantial changes in brain anatomy (Jäncke, 2009). Moreover, research over the last decade has provided strong evidence that these training-induced changes occur across the entire lifespan, from birth to old age (Seidler, 2007; Lustig et al., 2009).

The neuroanatomical underpinnings of proficient motor skills were initially studied in highly trained motor-experts (e.g., professional musicians), applying cross-sectional study designs. From these studies, one can conclude that skill acquisition or performance improvement induces specific structural changes in brain areas demanded by the practiced task (Gaser and Schlaug, 2003; Jäncke et al., 2009; Hänggi et al., 2010). Recent longitudinal neuroimaging studies focused on the impact of motor trainings lasting several days or weeks in untrained subjects and showed adaptations in both neural gray (Draganski et al., 2004; Driemeyer et al., 2008) and white (Scholz et al., 2009; Taubert et al., 2010) matter. However, longitudinal studies investigating training-induced changes in anatomical properties of the cortical

Received April 21, 2011; revised June 17, 2011; accepted July 20, 2011.

Author contributions: L.B., S.M., and L.J. designed research; L.B. and S.M. performed research; L.B., S.M., and C.G. analyzed data; L.B., S.M., C.G., and L.J. wrote the paper.

This work was supported by the Research Funding of the University of Zurich (56234103), a project grant from the Bundesamt für Sport (36234110), and the International Normal Aging and Plasticity Imaging Center, University of Zurich. L.B. is a fellow of the International Max Planck Research School on the Life Course.

The authors declare no competing interests.

Correspondence should be addressed to Ladina Bezzola, University of Zurich, Institute of Psychology/Neuropsychology, Binzmühlestrasse 14/25, CH-8050 Zurich, Switzerland. E-mail: I.bezzola@psychologie.uzh.ch.

DOI:10.1523/JNEUROSCI.1996-11.2011

Copyright $\odot 2011$ the authors $\quad 0270-6474 / 11 / 3112444-05 \$ 15.00 / 0$ motor system are rare, particularly in older age groups. As a consequence, there is little knowledge about the extent of the neural changes and factors influencing it, such as age (Boyke et al., 2008) or certain training characteristics (e.g., intensity or duration). In the motor domain, the existing longitudinal training studies in healthy subjects attached great importance to controlled training situations-keeping training parameters as constant as possible (e.g., training duration per day, overall training duration, training schedule, strategies, etc.). The neural effects of training performed without strict experimental control-for instance, as a leisure activity-have not yet been studied. Considering, however, that participation in leisure activities was shown to influence brain aging and the risk of developing dementia (Verghese et al., 2003; Colcombe et al., 2006), it is important to expand previous research by focusing on training that approximates the real-life situation and, therefore, possesses higher ecological validity.

Consequently, the present study was designed to explore training-induced neural adaptations in golf novices performing their regular golf practice in their specific golf clubs, without strict experimental control. Since our knowledge about neural plasticity is particularly limited in regards to middle-aged adults, we recruited subjects between the ages of 40 and 60 years. The main focus was on changes of neural gray matter, which can be ascribed to the golf practice. Given the complexity of learning to play golf, we expected a practice-induced gray matter increase in an extended neural network encompassing regions of the entire dorsal stream (Culham and Valyear, 2006) known to play a pivotal role in serving visuomotor skills, regions typically associated with motor learning (i.e., subcortical and cerebellar motor areas) as well as frontal associative regions concerned with more cognitive aspects of motor-skill acquisition (Doyon and Benali, 2005). 


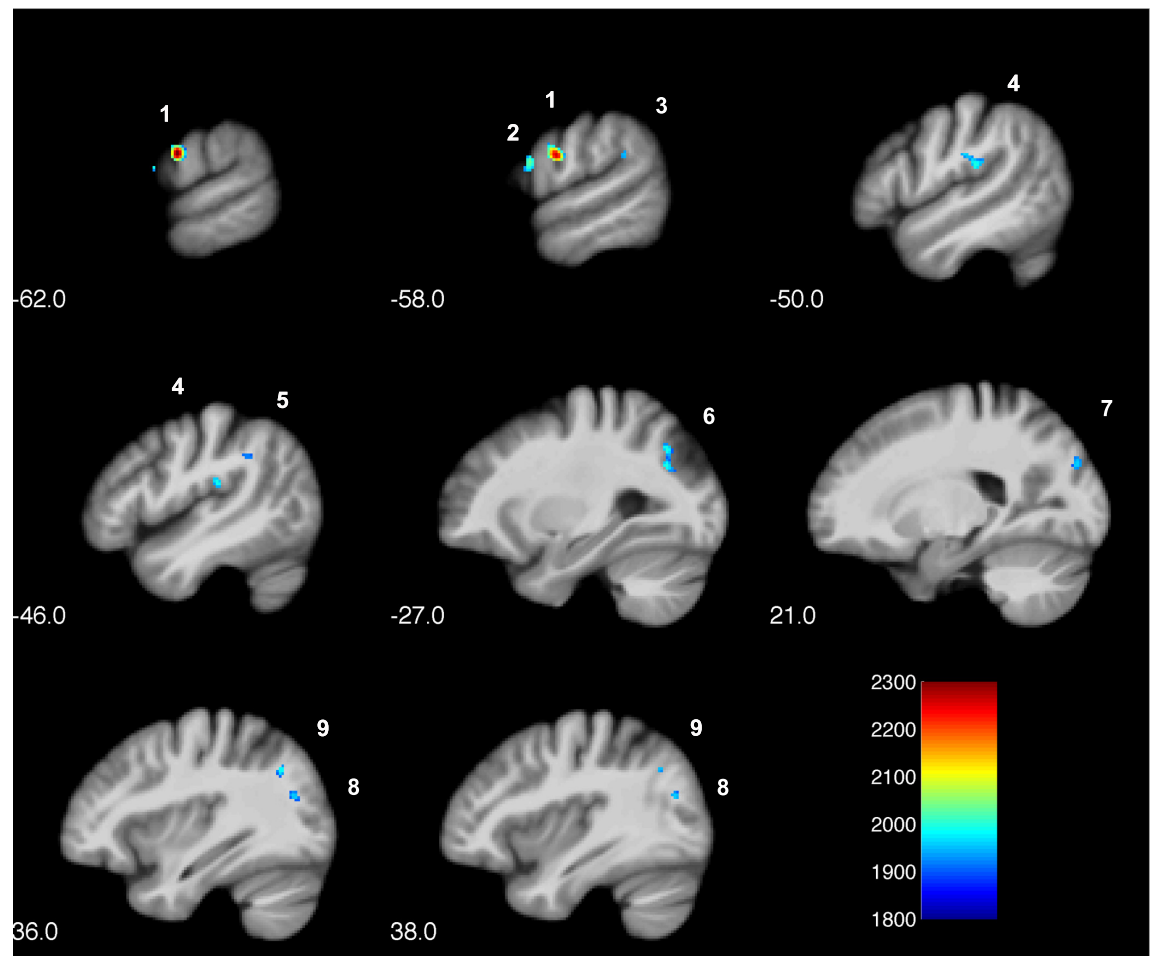

Figure 1. Gray matter increases (sagittal slices with $x$-coordinate value) in the golf group. 1, Central sulcus; 2 , ventral premotor cortex; 3, rostral inferior parietal lobule; 4, rostral inferior parietal lobule; 5 , rostral inferior parietal lobule; 6 , intraparietal sulcus; 7 , parieto-occipital junction; 8, caudal inferior parietal lobule; 9 , caudal inferior parietal lobule. The color bar represents the threshold-free cluster enhancement statistics.

\section{Materials and Methods}

Subjects. Twenty-two healthy volunteers (18 women, 4 men), with a mean age of $51.2(\mathrm{SD}=7.2)$ years participated in the present study. None of the participants had any history of neurological or psychiatric disorder. The study sample consisted of the following two groups, a golf novice group ( $n=11 ; 9$ women, 2 men) and an age- and sex-matched passive control group ( $n=11$; no specific training was applied in this group). Handedness was verified with the Annett handedness questionnaire (Annett, 1970). According to this test, 20 participants (10 golf group, 10 control group) were classified as consistent right-handers and two participants as consistent left-handers. Control participants were only included in the present study if they did not have any golf experience and did not start a new physical workout during the study period. All subjects gave written informed consent and the local ethics committee approved the present study, in compliance with the Helsinki Declaration.

Experimental design. We used a longitudinal study design with two measurement time points. At each measurement time point, a T1weighted image was recorded from each participant. The golf group had $\sim 77 \mathrm{~h}$ of golf pre-experience. The interval between the two measurements referred to the golf group and amounted to 40 golf-training hours. Each golf novice had an individual intermeasurement interval, calculated on the basis of the training hours and recorded on a training log. Accordingly, we inferred the training intensity (TI) from the elapsed time to achieve 40 training hours by using the following formula: $\mathrm{TI}=40 / x$ days, where $x$ is the number of days needed to reach a total of 40 training hours. Since each participant of the golf group was matched (age, sex, handedness) to a participant of the control group, we ensured that the two groups had the same intermeasurement interval.

MRI data acquisition. The MRI data were acquired on a 3T Philips Intera whole-body magnetic resonance tomograph equipped with an eight-channel Philips SENSE head coil. Whole-brain anatomical images were obtained from 160 slices by using a T1-weigthed three-dimensional gradient echo pulse sequence (turbo field echo). The following acquisition parameters were used: TR, $8 \mathrm{~ms}$; TE, $3.7 \mathrm{~ms}$; flip angle, $8^{\circ}$; FOV, $240 \times 240 ; 160$ slices; voxel size, $0.94 \times 0.94 \times 1 \mathrm{~mm}$.
Voxel-based morphometry. Data preprocessing and analysis was performed with the VBM8 toolbox (http://dbm.neuro.uni-jena.de/vbm/), which is incorporated in the SPM8 software (http://www.fil.ion.ucl.ac.uk/spm/) running on MATLAB R2010a (Mathworks). We applied the default longitudinal preprocessing approach integrated into the VBM8 toolbox. For this purpose, the following preprocessing steps were required: (1) registration of time point 2 (T2) scan to time point 1 (T1) scan for each subject separately, (2) intrasubject bias correction, (3) segmentation of the different tissue classes, (4) linear (i.e., affine) and nonlinear registration (i.e., Dartel), and (5) modulation of the different tissue segments by the nonlinear normalization parameters to account for individual brain size differences. The segmentation procedure was further refined by accounting for partial volume effects (Tohka et al., 2004), by applying adaptive maximum a posteriori estimations (Rajapakse et al., 1997), and by applying a hidden Markov randomfield model (Cuadra et al., 2005). Finally, the realigned and normalized gray matter segments were smoothed with an $8 \mathrm{~mm}$ FWHM Gaussian kernel.

Statistical analysis. First, we performed a repeated-measures ANOVA (i.e., flexible factorial model in SPM8) with withinsubject factor time (T1, T2) and betweensubject factor group (golf group, control group). We were particularly interested in increases and decreases in gray matter intensities of the golf group. To ascertain that gray matter increases seen in the golf group can exclusively be ascribed to the golf training, we performed an additional interaction analysis. This analysis was based on regions of interest (ROIs) extracted from the clusters showing a gray matter increase in the golf group.

Second, we performed an ROI analysis to analyze the trainingrelated structural changes in more detail. Accordingly, we created spherical ROIs with a radius of $8 \mathrm{~mm}$ by using the marsbar tool (http://marsbar.sourceforge.net/). The center coordinates were defined by the local maxima of the whole-brain analysis, i.e., the significantly increased gray matter regions of the golf group. For each golf participant and each ROI, we calculated the percentage increase in gray matter intensity. As a result, for each ROI, a regression analysis was performed with the predictor TI and the dependent variable percentage increase in gray matter.

Threshold-free cluster enhancement (Smith and Nichols, 2009) was used to detect significant clusters. This approach provides the ability to perform cluster-based inference without the need to initially specify a cluster-forming threshold. For each analysis, 5000 randomization runs were performed. Statistical maps were thresholded at $p<0.05$, corrected for the entire search volume.

\section{Results}

\section{Behavioral data}

The golf and control group did not differ in age and education (both $p>0.1$ ). The golf-training group needed a mean duration of $149.82 \mathrm{~d}$ (range, $92-235 \mathrm{~d}$ ) to achieve 40 training hours. Accordingly, the mean TI, defined as the ratio TI $=40 / x$ days, was 0.295 (SD = 0.096). Furthermore, participants who passed the basic entrance examination (License to Play on Golf Courses) successfully had practiced with a considerable higher intensity (Cohen's $d=2.60)$. Hence, this subgroup $(n=4)$ needed less time to achieve 40 training hours. 
Table 1. Significant gray matter changes in the golf group

\begin{tabular}{|c|c|c|c|c|c|c|c|}
\hline \multirow[b]{2}{*}{ Number } & \multirow[b]{2}{*}{ Anatomical area } & \multirow[b]{2}{*}{ Hemisphere } & \multicolumn{3}{|c|}{ MNI coordinates } & \multirow[b]{2}{*}{ Increase golf, $p<$} & \multirow[b]{2}{*}{ Interaction, $p<$ (Cohen's $d$ ) } \\
\hline & & & $x$ & $y$ & $z$ & & \\
\hline 1 & Central sulcus & $\mathrm{L}$ & -62 & -4 & 25 & 0.037 & $0.001(2.24)$ \\
\hline 2 & Ventral premotor cortex & $\mathrm{L}$ & -58 & 11 & 21 & 0.046 & $0.001(1.87)$ \\
\hline 3 & Rostral inferior parietal lobule & $\mathrm{L}$ & -58 & -43 & 25 & 0.049 & $0.001(1.62)$ \\
\hline 4 & Rostral inferior parietal lobule & $\mathrm{L}$ & -50 & -19 & 24 & 0.040 & $0.001(1.68)$ \\
\hline 5 & Rostral inferior parietal lobule & $\mathrm{L}$ & -46 & -45 & 33 & 0.043 & $0.003(1.47)$ \\
\hline 6 & Intraparietal sulcus & $\mathrm{L}$ & -27 & -67 & 28 & 0.050 & $0.012(1.18)$ \\
\hline 7 & Parieto-occipital junction & $\mathrm{R}$ & 21 & -85 & 30 & 0.046 & $0.001(1.64)$ \\
\hline 8 & Caudal inferior parietal lobule & $\mathrm{R}$ & 38 & -72 & 19 & 0.043 & $0.009(1.24)$ \\
\hline 9 & Caudal inferior parietal lobule & $\mathrm{R}$ & 36 & -63 & 34 & 0.042 & $0.380(0.38)$ \\
\hline
\end{tabular}

Significant gray matter changes in the golf group between the two measurement time points (increases in the golf group). All results are corrected for the entire search volume. The last column refers to the $p$ value (Bonferroni corrected) and the effect size (Cohen's $d$ ) of the interaction (Group $\times$ Time). The consecutive indices 1-9 relate to the numbers used in Figures 1 and 2.

\section{Main contrast: posttraining versus pretraining in the golf group}

In line with our hypotheses, the golf novices revealed increased gray matter in areas associated with the dorsal stream and critical regions in the process of motor learning (Fig. 1; Table 1). In the process of the golf training, increases were found in the ventral part of the central sulcus encompassing both the motor and sensory compartment (M1 and S1, respectively). A further region of gray matter increase is situated in the vicinity of the ventral premotor cortex (PMC) and the inferior frontal gyrus. Additionally, regions belonging to the left and right inferior parietal lobe showed training-induced increases in gray matter. In contrast, the other tests investigating differences between the two time points (i.e., decreases in the golf group, increases and decreases in the control group) and between the two groups at baseline did not differ with respect to gray matter.

\section{Interaction contrast: Group $\times$ Time Point}

The interaction analysis confirmed the increased gray matter changes of the golf group. While gray matter increases in the golf group were prominent in all tested regions, gray matter increases were absent or greatly reduced in the control group (Fig. 2). Table 1 contains the $p$ values [Bonferroni-corrected by applying the procedure suggested by Holm (1979)] and the effect sizes (Cohen's $d$ ) of the associated interaction effects. We would like to emphasize that all results remained stable in terms of effect sizes even when the two left-handers were excluded from the analyses.

\section{Regression analysis}

To assess the influence of training intensity on gray matter changes, we performed an ROI analysis. This analysis revealed a strong relationship $(r=0.83)$ between training intensity and percentage increase in the right parieto-occipital junction (POJ) (Fig. 3). As can be seen in Figure 3, there is a clear trend showing larger changes in this ROI in golf novices with a license to play golf courses compared with golf novices without this license (Cohen's $d=1.41$ ). Furthermore, we see a trend for a relationship between training intensity and percentage increase in the ventral $\operatorname{PMC}(r=0.56)$.

\section{Discussion}

Based on former research, we hypothesized that the golf novice group would demonstrate training-induced adaptations in gray matter in brain regions demanded by golf training. Two main findings of the present study provide support for this hypothesis. First, we show that $40 \mathrm{~h}$ of golf training induces an increase in gray matter in task-relevant cortical regions encompassing sensorimotor regions and areas belonging to the dorsal stream. Sec-

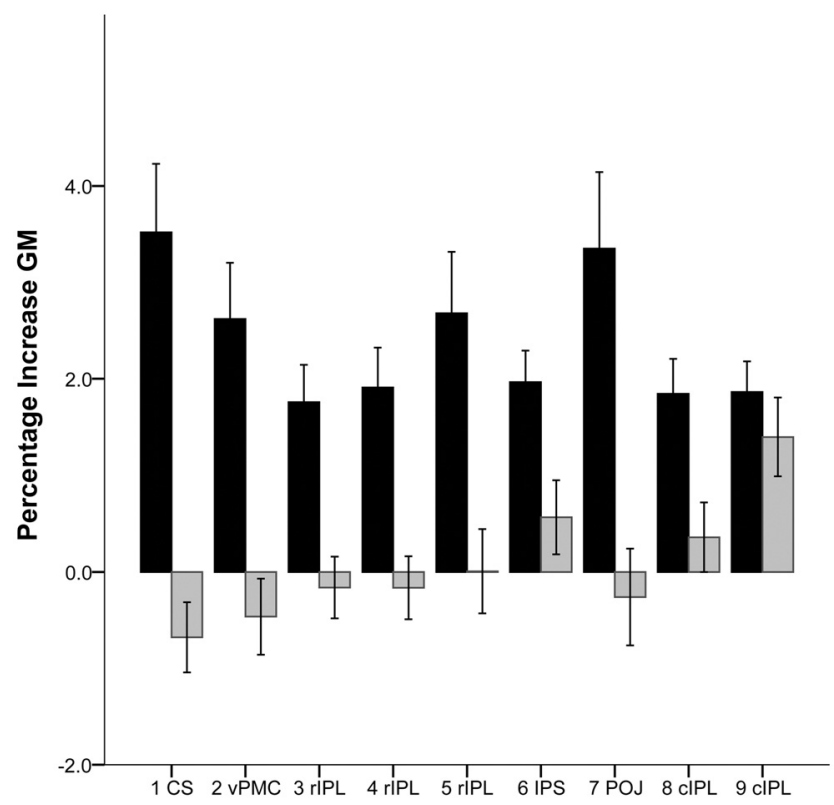

Figure 2. Interaction effects for changes in gray matter (GM) between the golf (black) and the control (gray) groups. CS, Central sulcus; vPMC, ventral premotor cortex; rIPL, rostral inferior parietal lobule; IPS, intraparietal sulcus; CIPL, caudal inferior parietal lobule. Error bars represent 1 SE. The indices $1-9$ relate to the numbers used in Figure 1 and Table 1.

ond, structural adaptations in the right POJ were strongly related to the intensity of training.

Our results are in good agreement with previous longitudinal studies demonstrating fast macroscopic changes of gray and white matter induced by a motor training (Driemeyer et al., 2008; Taubert et al., 2010). However, a new and striking result is that the training-induced gray matter changes observed in our study were not dependent on strict training or experimental constraints. Previous studies investigating training-induced neuronal plasticity attached great importance to keeping training parameters constant across subjects to optimize effect strengths (Lustig et al., 2009). Moreover, cross-sectional behavioral studies indicate a beneficial effect of certain leisure activities, such as dancing or playing chess, on the risk of developing dementia (Verghese et al., 2003; Rovio et al., 2005). Therefore, these studies point indirectly to neuronal adaptations resulting from being active during leisure time. To our best knowledge, our study is the first demonstrating robust neural effects induced by a true leisure activity, meaning that individuals of our training group had different training schedules and strategies depending on a variety of external factors, such as the time available for leisure activities or 


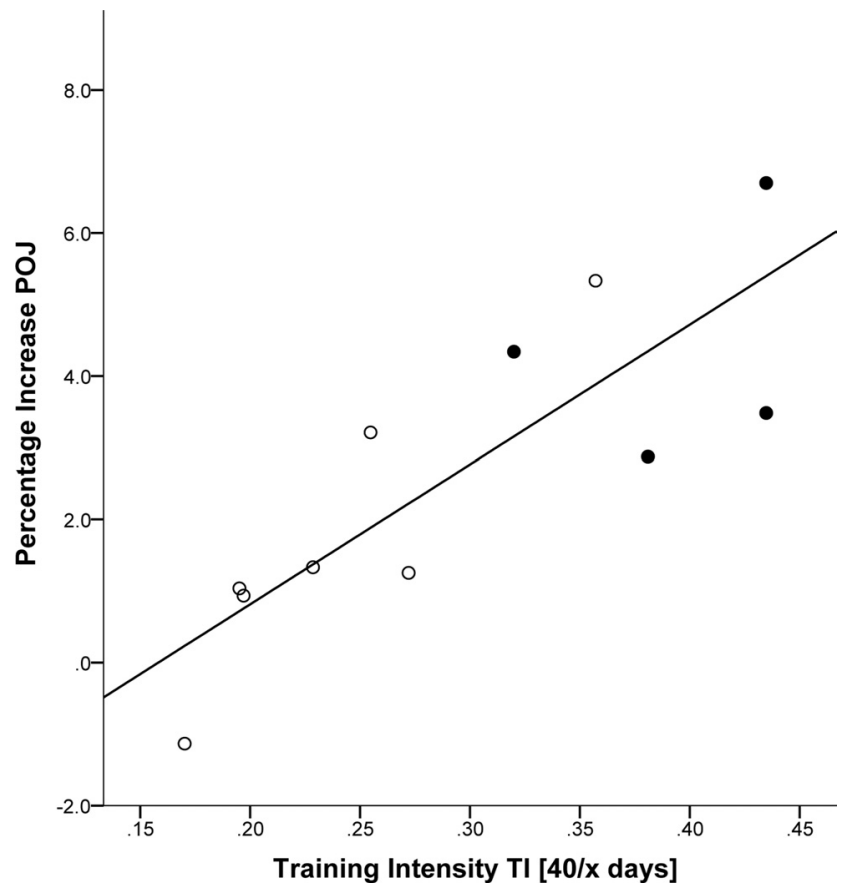

Figure 3. Correlation of gray matter percentage increase in the POJ and TI. Filled dots represent golf participants who passed the basic entrance examination within the study period.

the teaching style of their individual golf instructor. Thus, the present study expands our knowledge about training-induced neuroplasticity by demonstrating effects of motor training with an improved ecological validity.

The process of learning to play golf comprises adaptations in motor functions, visuospatial functions, and body perception/ control. These functions correspond well to regions showing training-related gray matter changes. First, the ventral PMC has been repeatedly associated with mental imagination and movement observation (Binkofski and Buccino, 2006; Caspers et al., 2010), both of which represent important strategies of motor skill learning, especially in the early stages of the learning process. Movement observation, in turn, is closely related to movement imitation-relying on the mirror neuron circuit, including the inferior frontal gyrus, the ventral PMC, and the inferior parietal cortex (Buccino et al., 2004). Concerning the observed adaptation in the vicinity of the dorsal and ventral PMC, the primary somatosensory cortex, and the inferior frontal gyrus, the generative assembly device (GAD) may be regarded as an alternative explanation (Corballis, 1991). The GAD proposed by Corballis (1991) suggests that the above-mentioned areas may be involved in the transformation of single elementary movements into a complex movement representation. In particular, at an early stage of learning, the incorporation of single movements to execute a complete golf swing is of great importance. Although the GAD is based mainly on language processes, a great amount of work has substantiated that this device also includes the motor system (Baumgaertner et al., 2007; Rizzolatti and Fabbri-Destro, 2010).

Both lines of research - motor learning and mirror neuronshave emphasized the functional connection between frontal regions (i.e., ventral PMC, inferior frontal gyrus) and the parietal lobe, which is based on extensive anatomical connections (Rushworth et al., 2006). Accordingly, we found training-induced changes in rostral and caudal parts of the inferior parietal lobe (IPL). The supramarginal gyrus, which is part of the rostral infe- rior parietal lobe, has been suggested to be important in planning goal-oriented actions (Tunik et al., 2008), particularly with respect to the integration of multimodal information (Eickhoff et al., 2010). Proprioceptive and tactile but also visual information needs to be precisely integrated before the execution of a broad but accurate arm movement. Consistent with this idea, the rostral part of the IPL was shown to be involved in perceiving one's own body (i.e., body ownership) (Blanke et al., 2005; Tsakiris et al., 2010). In contrast, the caudal part of the IPL and the POJ are functionally and anatomically connected to the visual system (Colby and Goldberg, 1999; Culham and Valyear, 2006). The increased gray matter density in the caudal part of the inferior parietal cortex is in agreement with previous studies investigating structural changes induced by complex motor training (i.e., juggling) (Draganski et al., 2004; Scholz et al., 2009). Juggling and golf share a variety of similar demands, especially a precise transformation from visual stimuli into a motor command and coding of spatial information.

Interestingly, in the present study, we demonstrate a strong positive relationship between the increase in gray matter and training intensity in the right POJ, a critical structure of the dorsal stream. Moreover, participants who practiced with a higher intensity (needed fewer days to complete the 40 training hours) and showed greater structural adaptation in the POJ were those achieving the license to play on the golf course within the 40 practice hours. Although we are aware that a strict assignment of a given function to a certain structure would undervalue the great complexity and connectivity of the human brain, previous research (i.e., animal studies, lesion studies, neuroimaging studies) provides convincing evidence that the POJ is closely associated with visuomotor processes, particularly in the on-line control and on-line correction of visually guided arm movements (Himmelbach et al., 2006; Kravitz et al., 2011). In general, our findings suggest that low- and high-intensity trainings are accompanied by a change of neuroanatomical parameters. Beyond that, we provide evidence that, in certain regions, differences between subjects practicing with low- or high-intensity can be observed. However, this latter finding must be interpreted with caution for two reasons. First, in our relatively small sample, subjects practicing with high intensity were also those showing a better performance (in terms of achieving the license to play on golf courses). Thus, the extent of gray matter change may also be directly linked with a certain level of proficiency or with an increase in proficiency. In this case, the relationship is likely moderated by factors (e.g., motivation, prior experience in other motor skills) favoring a faster skill acquisition within the same amount of training hours. Future studies are needed to shed more light on this issue. Second, we demonstrated a strong relationship between structure and behavior only in the POJ. The lack of correlations between training intensity and increases in gray matter in regions other than the POJ may be due to the number of subjects in the golf group, which is at the lower limit for revealing brain-behavior correlations. Thus, we cannot rule out the possibility that the statistical power was too low to detect such correlations for further cortical/subcortical regions. Alternatively, it may be that the increase of gray matter volume in regions other than POJ is independent of training intensity, but instead depends on the total amount of training hours between premeasurements and postmeasurements, which was the same for all subjects.

Generally, however, it can be concluded that changes in gray matter are closely related to skill level, which in turn depends in large part on training characteristics. Thus, subjects who prac- 
ticed with higher intensity and reached the license to play on golf courses within the studied training interval are assumed to quickly run through the successive handicap levels in the future (provided that training intensity does not decline)-a process that is likely accompanied by a further increase of gray matter. Comparing them to subjects who practiced with lower intensity and who are assumed to run through the handicap levels much slower, one would expect between-group differences in gray matter at a given point in time, which would resemble previous findings of our group showing cross-sectional differences between highly trained and amateur golfers (Jäncke et al., 2009). The present study extends findings from our previous study by showing that structural adaptations due to golf practice can be observed as early as immediately after the onset of golf training when using a longitudinal approach, which has a higher statistical power.

The results described above are intriguing because they demonstrate that a physical leisure activity practiced with an individual training protocol revealed very similar effects as a more experimentally controlled training. Thus, we assume that a strict and controlled training protocol is not mandatory for traininginduced adaptations of gray matter. Beyond this, our findings indicate an interesting relationship between training intensity and the extent of training-induced gray matter change. However, future research needs to clarify whether this relationship is exclusively due to training characteristics or, for example, due to the higher level of proficiency that accompanies - in case of our subjects-a higher training intensity. Lastly, regarding the practical implications of our findings, we propose that leisure activities performed with low to moderate intensity evoke anatomical changes similar to those evoked by highly controlled trainings in an experimental setting. Thus, leisure activities may be considered an additional therapeutic setting in the process of neuro-rehabilitation.

\section{References}

Annett M (1970) A classification of hand preference by association analysis. Br J Psychol 61:303-321.

Baumgaertner A, Buccino G, Lange R, McNamara A, Binkofski F (2007) Polymodal conceptual processing of human biological actions in the left inferior frontal lobe. Eur J Neurosci 25:881-889.

Binkofski F, Buccino G (2006) The role of ventral premotor cortex in action execution and action understanding. J Physiol Paris 99:396-405.

Blanke O, Mohr C, Michel CM, Pascual-Leone A, Brugger P, Seeck M, Landis T, Thut G (2005) Linking out-of-body experience and self processing to mental own-body imagery at the temporoparietal junction. J Neurosci 25:550-557.

Boyke J, Driemeyer J, Gaser C, Büchel C, May A (2008) Training-induced brain structure changes in the elderly. J Neurosci 28:7031-7035.

Buccino G, Vogt S, Ritzl A, Fink GR, Zilles K, Freund HJ, Rizzolatti G (2004) Neural circuits underlying imitation learning of hand actions: an eventrelated fMRI study. Neuron 42:323-334.

Caspers S, Zilles K, Laird AR, Eickhoff SB (2010) ALE meta-analysis of action observation and imitation in the human brain. Neuroimage 50:1148-1167.

Colby CL, Goldberg ME (1999) Space and attention in parietal cortex. Annu Rev Neurosci 22:319-349.

Colcombe SJ, Erickson KI, Scalf PE, Kim JS, Prakash R, McAuley E, Elavsky S, Marquez DX, Hu L, Kramer AF (2006) Aerobic exercise training increases brain volume in aging humans. J Gerontol A Biol Sci Med Sci 61:1166-1170.

Corballis MC (1991) The lopsided ape: evolution of the generative mind. New York: Oxford UP.

Cuadra MB, Cammoun L, Butz T, Cuisenaire O, Thiran JP (2005) Compar- ison and validation of tissue modelization and statistical classification methods in T1-weighted MR brain images. IEEE Trans Med Imaging 24:1548-1565.

Culham JC, Valyear KF (2006) Human parietal cortex in action. Curr Opin Neurobiol 16:205-212.

Doyon J, Benali H (2005) Reorganization and plasticity in the adult brain during learning of motor skills. Curr Opin Neurobiol 15:161-167.

Draganski B, Gaser C, Busch V, Schuierer G, Bogdahn U, May A (2004) Neuroplasticity: changes in grey matter induced by training. Nature 427:311-312.

Driemeyer J, Boyke J, Gaser C, Büchel C, May A (2008) Changes in gray matter induced by learning-revisited. PLoS One 3:e2669.

Eickhoff SB, Jbabdi S, Caspers S, Laird AR, Fox PT, Zilles K, Behrens TE (2010) Anatomical and functional connectivity of cytoarchitectonic areas within the human parietal operculum. J Neurosci 30:6409-6421.

Gaser C, Schlaug G (2003) Brain structures differ between musicians and non-musicians. J Neurosci 23:9240-9245.

Hänggi J, Koeneke S, Bezzola L, Jäncke L (2010) Structural neuroplasticity in the sensorimotor network of professional female ballet dancers. Hum Brain Mapp 31:1196-1206.

Himmelbach M, Karnath HO, Perenin MT, Franz VH, Stockmeier K (2006) A general deficit of the 'automatic pilot' with posterior parietal cortex lesions? Neuropsychologia 44:2749-2756.

Holm S (1979) A simple sequentially rejective multiple test procedure. Scand J Statist 6:65-70.

Jäncke L (2009) The plastic human brain. Restor Neurol Neurosci 27: 521-538.

Jäncke L, Koeneke S, Hoppe A, Rominger C, Hänggi J (2009) The architecture of the golfer's brain. PLoS One 4:e4785.

Kravitz DJ, Saleem KS, Baker CI, Mishkin M (2011) A new neural framework for visuospatial processing. Nat Rev Neurosci 12:217-230.

Lustig C, Shah P, Seidler R, Reuter-Lorenz PA (2009) Aging, training, and the brain: a review and future directions. Neuropsychol Rev 19:504-522.

Rajapakse JC, Giedd JN, Rapoport JL (1997) Statistical approach to segmentation of single-channel cerebral MR images. IEEE Trans Med Imaging $16: 176-186$.

Rizzolatti G, Fabbri-Destro M (2010) Mirror neurons: from discovery to autism. Exp Brain Res 200:223-237.

Rovio S, Kåreholt I, Helkala EL, Viitanen M, Winblad B, Tuomilehto J, Soininen H, Nissinen A, Kivipelto M (2005) Leisure-time physical activity at midlife and the risk of dementia and Alzheimer's disease. Lancet Neurol 4:705-711.

Rushworth MF, Behrens TE, Johansen-Berg H (2006) Connection patterns distinguish 3 regions of human parietal cortex. Cereb Cortex 16:1418-1430.

Scholz J, Klein MC, Behrens TE, Johansen-Berg H (2009) Training induces changes in white-matter architecture. Nat Neurosci 12:1370-1371.

Seidler RD (2007) Older adults can learn to learn new motor skills. Behav Brain Res 183:118-122.

Smith SM, Nichols TE (2009) Threshold-free cluster enhancement: addressing problems of smoothing, threshold dependence and localisation in cluster inference. Neuroimage 44:83-98.

Taubert M, Draganski B, Anwander A, Müller K, Horstmann A, Villringer A, Ragert P (2010) Dynamic properties of human brain structure: learning-related changes in cortical areas and associated fiber connections. J Neurosci 30:11670-11677.

Tohka J, Zijdenbos A, Evans A (2004) Fast and robust parameter estimation for statistical partial volume models in brain MRI. Neuroimage 23:84-97.

Tsakiris M, Longo MR, Haggard P (2010) Having a body versus moving your body: neural signatures of agency and body-ownership. Neuropsychologia 48:2740-2749.

Tunik E, Lo OY, Adamovich SV (2008) Transcranial magnetic stimulation to the frontal operculum and supramarginal gyrus disrupts planning of outcome-based hand-object interactions. J Neurosci 28:14422-14427.

Verghese J, Lipton RB, Katz MJ, Hall CB, Derby CA, Kuslansky G, Ambrose AF, Sliwinski M, Buschke H (2003) Leisure activities and the risk of dementia in the elderly. N Engl J Med 348:2508-2516. 